\title{
Turbulent entrainment origin of protostellar outflows
}

\author{
Guang-Xing $\mathrm{Li}^{1}$, Keping Qiu ${ }^{1,2}$, Friedrich Wyrowski ${ }^{1}$, and Karl Menten ${ }^{1}$ \\ 1 Max-Planck Institut für Radioastronomie, Auf dem Hügel, 69, 53121 Bonn, Germany \\ e-mail: gxli@mpifr-bonn.mpg.de \\ 2 School of Astronomy and Space Science, Nanjing University, Nanjing 210093, PR China
}

Received 17 October 2012 / Accepted 27 August 2013

\section{ABSTRACT}

\begin{abstract}
Protostellar outflow is a prominent process that accompanies the formation of stars. It is generally agreed that wide-angled protostellar outflows come from the interaction between the wind from a forming star and the ambient gas. However, it is still unclear how the interaction takes place. In this work, we theoretically investigate the possibility that the outflow results from interaction between the wind and the ambient gas in the form of turbulent entrainment. In contrast to the previous models, turbulent motion of the ambient gas around the protostar is taken into account. In our model, the ram-pressure of the wind balances the turbulent ram-pressure of the ambient gas, and the outflow consists of the ambient gas entrained by the wind. The calculated outflow from our modelling exhibits a conical shape. The total mass of the outflow is determined by the turbulent velocity of the envelope as well as the outflow age, and the velocity of the outflow is several times higher than the velocity dispersion of the ambient gas. The outflow opening angle increases with the strength of the wind and decreases with the increasing ambient gas turbulence. The outflow exhibits a broad line width at every position. We propose that the turbulent entrainment process, which happens ubiquitously in nature, plays a universal role in shaping protostellar outflows.
\end{abstract}

Key words. stars: winds, outflows - stars: massive - ISM: jets and outflows - ISM: kinematics and dynamics - turbulence line: profiles

\section{Introduction}

Protostellar outflow is a prominent process that is intimately connected with the formation of stars. When a protostar accretes gas from its surroundings, it produces a powerful wind or jet. As the wind or jet material moves away from the protostar, ambient gas is entrained. The mixture of the wind or jet gas and the ambient gas will move away from the protostar, forming a so-called molecular outflow. In observations of the star-forming regions with rotational transitions of molecules such as $\mathrm{CO}$, outflows can be easily identified from the high-velocity part of the emission line. The physical size of the molecular outflow is about a parsec. The typical velocity of the outflowing gas is about tens of kilometers per second, and the morphology of the outflow can be collimated (jet-like) or less collimated (conical-shaped), or a combination of both.

Many of the outflows exhibit as conical-shaped geometry (Shepherd et al. 1998; Lee et al. 2000; Arce \& Sargent 2006; Qiu et al. 2009; Ren et al. 2011; Cyganowski et al. 2011). It is still unclear how these conical-shaped outflows form. Models that can explain conical-shaped outflows include the wind-driven shell model (Shu et al. 1991; Li \& Shu 1996; Lee et al. 2001) and the circulation model (Fiege \& Henriksen 1996; Lery et al. 1999). In the wind-driven shell model, the wind from the protostar blows into the ambient medium, and a shell forms at the interface between the wind and the ambient gas. The shell absorbs momentum from the wind and expands. The outflowing material consists of the ambient gas swept up by the shell. In the circulation model, the gas circulates around the protostar in a quadrupolar way, and it is the gas that moves outward in this circulation cycle that constitutes the outflow.
Neither of these models properly addresses the role of supersonic turbulent motion in the formation and evolution of the protostellar outflows. This might be because the importance of supersonic turbulent motion in star-forming regions was not generally recognized when these models were proposed. Today, it is widely recognized that the interstellar medium where stars form is turbulent, this turbulence is supersonic in most star-forming regions (Larson 1981; Ballesteros-Paredes et al. 2007). This will bring about serious physical discrepancies to both models. For the wind-driven shell model, the turbulent ram-pressure (expressed as $p_{\text {turb }} \sim \rho \sigma_{\text {turb }}^{2}$ ) is much higher than the thermal pressure, and is often comparable to the ram-pressure of the wind (expressed as $p_{\text {wind }} \sim \rho_{\text {wind }} v_{\text {wind }}^{2}$, see Sect. 4). Therefore the wind-driven shell model is no longer valid since the physical condition cannot allow the shell to expand for long. The circulation model is also questionable when the interstellar medium is turbulent, because turbulent motion is expected to significantly distort the motion of gas, making the initial condition unfavourable for circulation to occur.

The kinematic structures of the outflows are only poorly reproduced by the previous models. Mappings of outflows with molecular lines reveal the spatial and velocity structures of the outflowing gas, which is crucial to test the models. It is found that the outflows ubiquitously exhibit a broad line width at different positions. Seen from position-velocity diagrams, the outflowing gas exhibits a wide velocity spread at one given position (Sect. 3). This is difficult to understand in the context of the widely accepted wind-driven shell model (Shu et al. 1991; Li \& Shu 1996; Lee et al. 2001), because the shell swept by the wind will expand according to the Hubble law, and the expansion speed of the shell is higher in the directions where the shell 
has progressed further. Seen from the position-velocity diagram, we can identify two velocity components at one given position. One component comes from the front wall of the outflow cavity, the other from the back wall of the outflow cavity (Lee et al. 2000). However, observations found that at one single position, there is only one broad component (e.g. Qiu et al. 2009).

It is observationally found that the opening angles of outflows evolve significantly during their lifetimes (Beuther \& Shepherd 2005; Arce \& Sargent 2006). While usually interpreted as the evolution of the wind-envelope interaction (Arce $\&$ Sargent 2006), a comprehensive treatment of this interaction in the presence of the turbulent motion of the ambient gas is still lacking.

In this study, we theoretically investigate the formation of outflows from the turbulent mixing between the wind from the protostar and the turbulent ambient gas. In our wind-driven turbulent entrainment model, the ram-pressure of the wind and the turbulent ram-pressure of the ambient gas (envelope) establish hydrostatic balance, and the outflow is the gas contained in the turbulent entrainment layer between the wind and the turbulent envelope. In Sect. 2 we present the basic physical picture, followed by a detailed description of our model. In Sect. 3 we compare the image and position-velocity diagram from our model with existing observations. In Sects. 4.1 and 4.2 we offer a unified framework to understand outflows from low-mass protostars and high-mass protostars as well as AGN-driven outflows. We also discuss the possibility that the wind is not strong enough to push the envelope, and predict the existence of dwarf outflows in these situations (Sect. 4.3). In Sect. 5 we conclude.

Recently, the interaction between a wind and a turbulent core has been studied with radiation-hydrodynamic simulations (Offner et al. 2011), focusing on how the physical structure suggested by the simulations can be reproduced through millimetrewavelength aperture synthesis observations. In this work, we present an explicit treatment of the mixing entrainment process, and study the consequences of this turbulent entrainment in producing protostellar outflows.

\section{Model}

In our model, the outflowing gas is contained in the turbulent entrainment layer that develops between the wind and the envelope (Fig. 1). The wind is collimated, and its strength reaches a maximum around the $z$-axis of Fig. 1. The wind is launched in a region close to the protostar due to magnetic centrifugal forces. This wind is generally collimated and exhibits universal asymptotic collimation properties (Shu et al. 1995; Matzner \& McKee 1999). The wind evacuates a cavity and is confined mainly inside it. The ambient matter (envelope) is still present in regions father away from the outflow axis. The outflow layer lies between the cavity and the ambient matter. The outflow is generated by the interaction between the wind and the envelope.

The turbulent nature of the interstellar medium has been recognized for years. It has been suggested that it plays an important role in regulating the star formation processes inside molecular clouds (Plume et al. 1997; Krumholz \& McKee 2005; Ballesteros-Paredes et al. 2007). Molecular outflows have been suggested as major drivers of this turbulent motion (Matzner 2007; Nakamura \& Li 2007). The role of turbulence in the formation and evolution of the molecular outflow remains unexplored. It is easy to show (Sect. 4.1) that at physical scales of about a parsec (which is the typical physical scale of the outflow), turbulence dominates the dynamical properties of the

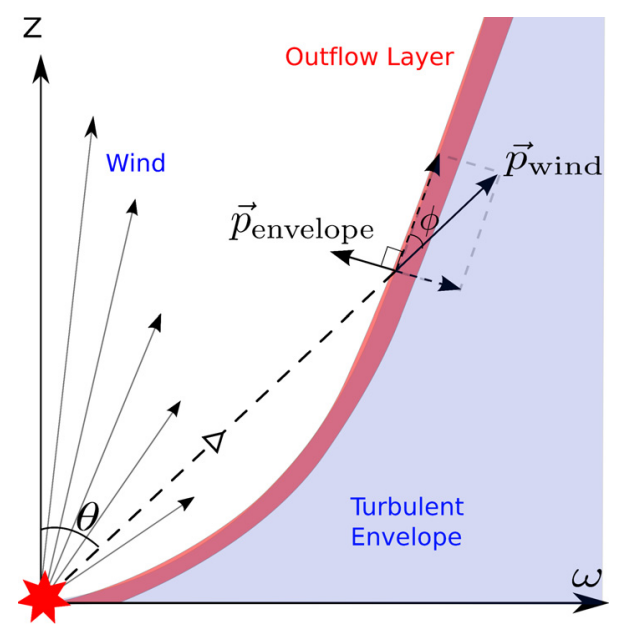

Fig. 1. Cartoon showing the basic structure of our model. The outflow (red region) lies between the wind and the envelope. Arrows ( $\boldsymbol{p}_{\text {wind }}$ and $\left.p_{\text {envelope }}\right)$ denote the ram-pressure of the wind and the envelope. With wind we denote the cavity evacuated by the wind, with turbulent envelope we denote the turbulent envelope, and with outflow layer we denote the layer which contains the mixture of gas from the wind and gas from the envelope.

medium. It is therefore necessary to consider the roles of turbulence consistently.

In this study, we propose that the structure of the outflow is determined by the following processes: first, we propose that the wind and the envelope will establish hydrostatic balance, and this balance will determine the shape of the outflow. According to Fig. 1, the wind has a ram-pressure that points away from the protostar (see $\boldsymbol{p}_{\text {wind }}$ in Fig. 1 , where $\left|\boldsymbol{p}_{\text {wind }}\right| \equiv p_{\text {wind }}=\rho_{\text {wind }} v_{\text {wind }}^{2}$ ), and the envelope has a ram-pressure that is perpendicular to the wall of the outflow cavity ( $\boldsymbol{p}_{\text {envelope }}$ ). According to the balance of these two forces, the shape of the outflow cavity can be numerically calculated (Sect. 2.2).

Second, we propose that a turbulent mixing layer develops between the wind and the envelope. When the wind moves relative to the envelope, Kelvin-Helmholtz instability occurs. When the instability saturates, a mixing layer establishes. This mixing layer grows as it absorbs mass and momentum from the envelope and the wind. The mass and momentum of the mixing layer is determined by mass and momentum conservations (Sects. 2.3, 2.4).

Turbulence plays an important role in both processes. First, turbulence influences the turbulent ram-pressure of the envelope, and in turn influences the pressure balance between the wind and the envelope. Therefore, turbulence influences the shape of the outflow (Sect. 2.2). Second, turbulence changes the mixing process that occurs between the wind and the envelope. Therefore, it influences the mass and velocity of the outflow (Sects. 2.3, 2.4).

The structure of the molecular cloud is inhomogeneous at a variety of scales. On a parsec scale, millimeter/sub-millimeter studies suggest that the density distribution of molecular gas around the protostar is consistent with being spherically symmetric and can be described as a power-law $\rho \sim r^{\gamma}$ (e.g. Keto \& Zhang 2010; Longmore et al. 2011).

Following these author we parametrized the density distribution of the envelope as

$\frac{\rho}{\rho_{0}}=\left(\frac{r}{r_{0}}\right)^{-k_{\rho}}$. 
Table 1. Summary of model parameters.

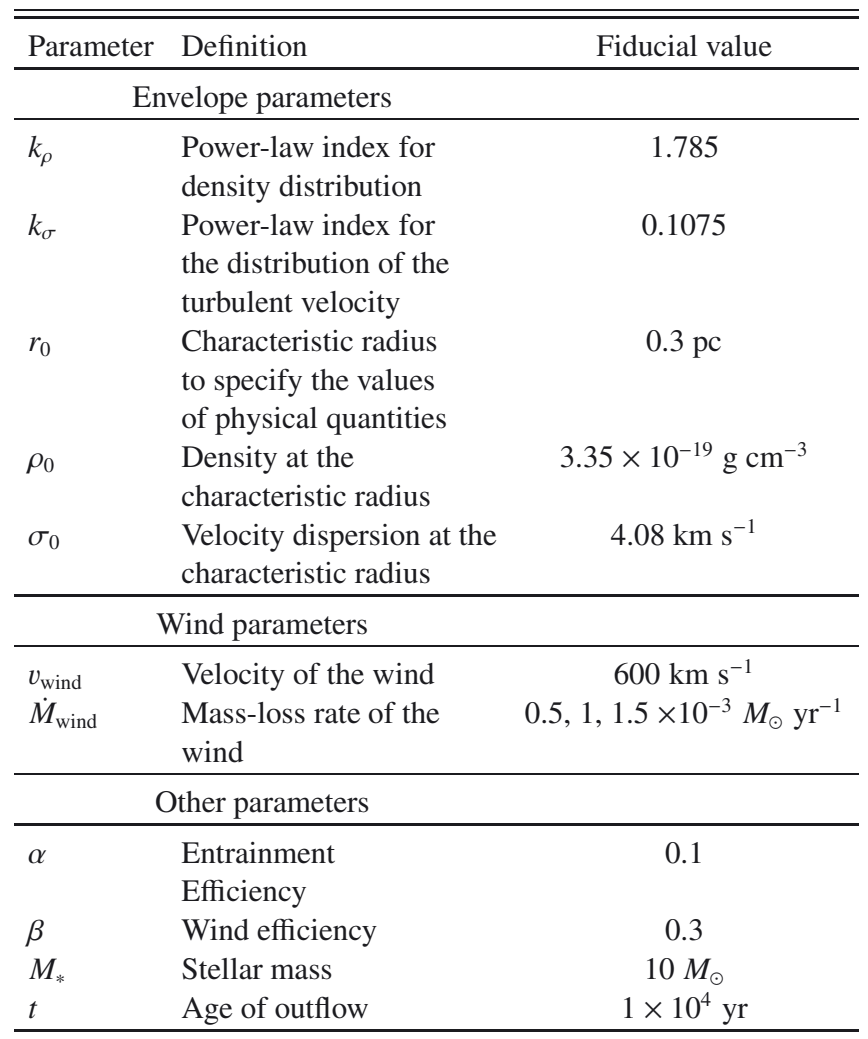

By assuming hydrostatic equilibrium, Eq. (1) leads to the distribution of the turbulent velocity $\sigma_{\text {turb }}$ that takes the form (McLaughlin \& Pudritz 1996; McKee \& Holliman 1999; McKee \& Tan 2003)

$\frac{\sigma_{\text {turb }}}{\sigma_{0}}=\left(\frac{r}{r_{0}}\right)^{1-k_{\rho} / 2}$.

The power-law index $k_{\rho}$ in Eq. (1) is set according to the observational studies by Beuther et al. (2002), Mueller et al. (2002), Keto \& Zhang (2010), Longmore et al. (2011). In our canonical model, consulting the results in Longmore et al. (2011) we set a density of $3.35 \times 10^{-19} \mathrm{~g} \mathrm{~cm}^{-3}$ at $r=0.3 \mathrm{pc}$ for Eq. (1). The parameter values are summarized in Table 1 . These parameters are set to be typical of high-mass protostars (e.g. Keto \& Zhang 2010; Longmore et al. 2011). Furthermore, since our model is scale-free, the results can be scaled to different situations (Sect. 4.4)

\subsection{Wind from the embedded protostar}

In our model, the outflow consists of the envelope gas entrained by the wind. To specify the wind, we followed the formalism used by Lee et al. (2001). In a spherical coordinate system $(r, \theta, \phi)$, Shu et al. (1995) have shown that the radial velocity of an $\mathrm{X}$-wind is roughly constant, and the density distribution takes the form

$\rho_{\text {wind }} \sim \frac{1}{r^{2} \sin ^{2} \theta}$.

Equation (3) is shown to be valid not only for the $\mathrm{X}$-wind model, but also for more general force-free winds (Ostriker 1997; Matzner \& McKee 1999). It has been shown that the terminal velocity of the wind $v_{\text {wind }}$ is approximately the same at different angles (Najita \& Shu 1994). In our modelling, the wind is parametrized as

$$
\begin{aligned}
& \rho_{\text {wind }}=\rho_{\text {norm }} \frac{1}{r^{2}\left(\sin ^{2} \theta+\epsilon\right)} \\
& v_{\text {wind }}=v_{t},
\end{aligned}
$$

where $\epsilon=0.01$ is added to avoid the singularity at the pole $(\theta=0)$ (Lee et al. 2001). $v_{\mathrm{t}}$ is the terminal velocity of the wind, and $\rho_{\text {norm }}$ is related to the wind mass-loss rate (rate at which the wind carries matter from the protostar, $\dot{M}_{\text {wind }}$ ) by mass conservation,

$\int_{0}^{\pi} 2 \pi r^{2} \sin \theta \rho_{\text {wind }}(r) v_{\text {wind }} \mathrm{d} \theta=\dot{M}_{\text {wind }}$.

We assumed that the wind has a terminal velocity of $600 \mathrm{~km} \mathrm{~s}^{-1}$ (similar to Cunningham et al. 2005). The values for the massloss rate were chosen to match the values found by Beuther et al. (2004). Parameters for the wind are listed in Table 1.

In principle, both the mass-loss rate of the wind and the density distribution of the envelope should evolve during the star formation process. However for simplicity, we only considered an envelope with a fixed density distribution and a wind with a fixed mass-loss rate. In practice, the timescale for local collapse is proportional to $\rho^{-1 / 2}$. Therefore, the central region where the protostars form evolves faster than the outer envelope, the density structure of the envelope evolves more slowly than the evolution of the wind mass-loss rate during the lifetime of the outflow.

\subsection{Shape of the outflow cavity}

The shape of the outflow cavity is determined by the balance of the ram-pressure of the wind and the pressure of the envelope perpendicular to the outflow cavity wall (see Fig. 1). The magnitude of $\boldsymbol{p}_{\text {wind }}$ is $p_{\text {wind }}=\rho_{\text {wind }} v_{\text {wind }}^{2}$, and the magnitude of the rampressure vector $\boldsymbol{p}_{\text {envelope }}$ is $p_{\text {envelope }}=\rho_{\text {envelope }} \sigma_{\text {turb }}^{2}$. Projected perpendicular to the edge of the outflow cavity, the magnitude of the wind pressure is $\left|\boldsymbol{p}_{\text {wind }}\right| \sin \phi$ and the magnitude of the envelope pressure is $\left|\boldsymbol{p}_{\text {envelope }}\right|$ (Fig. 1). The pressure balance perpendicular to the wall of the outflow cavity can then be written as

$p_{\text {wind }} \sin \phi=p_{\text {envelope }}$.

Along the cavity wall, no pressure balance is established, and the wind contributes its momentum to the outflow (see Sect. 2.3).

Here we used cylindrical coordinates $(\omega, \phi, z)$ for convenience. The polar angle $\theta$ is specified as (Fig. 1)

$\theta=\frac{\pi}{2}-\arctan \left(\frac{z}{\omega}\right)$,

and the angle between the cavity wall and the $z$ axis is specified as

$\theta_{\mathrm{wall}}=\frac{\pi}{2}-\arctan \left(\frac{\mathrm{dz}}{\mathrm{d} \omega}\right)$.

This gives a value $\phi$ of (Fig. 1)

$\phi=\theta-\theta_{\text {wall }}=\arctan \left(\frac{\mathrm{dz}}{\mathrm{d} \omega}\right)-\arctan \left(\frac{z}{\omega}\right)$.

Combining Eqs. (6)-(9) gives

$\frac{\mathrm{dz}}{\mathrm{d} \omega}=\tan \phi=\tan \left(\arctan \left(\frac{z}{\omega}\right)+\arcsin \frac{p_{\text {envelope }}}{p_{\text {wind }}}\right)$. 


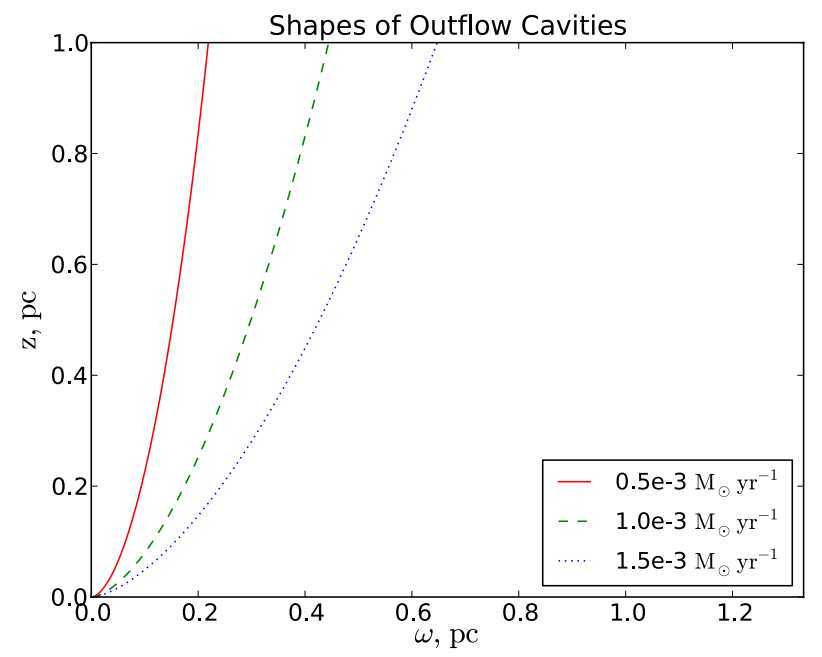

Fig. 2. Shapes of outflow cavities calculated for models with different wind mass-loss rates. The parameters are taken from Table 2. See Sect. 2.2 for details.

Equation (10) determines the shape of the outflow cavity. It is a first-order differential equation and can be solved numerically. We imposed the boundary condition $\omega=4.5 \times 10^{15} \mathrm{~cm}$ at $z=0$, which is similar to those used by Parkin et al. (2009). The results are not sensitive to the choice of this boundary condition.

In deriving Eq. (10), we have neglected the effect of centrifugal force. As will be shown in Appendix A, centrifugal force is not significant in our case.

Using Eq. (10), we calculated the shapes of the outflow cavities for winds of different mass-loss rates. The results are shown in Fig. 2. It is clear that the opening angle of the outflow depends on the strength of the wind: a stronger wind leads to a less collimated outflow.

Observationally, the opening angle of the outflow evolves during the evolution of the protostar (Arce \& Sargent 2006; Beuther \& Shepherd 2005). This is interpreted as the evolution of the wind-envelope interaction (Arce \& Sargent 2006). However, according to the widely accepted wind-driven shell model, the opening angle of the outflow should be fixed as the wind collimation is fixed and the density structure of the envelope does not evolve much (Lee et al. 2001). How the evolution of the wind-envelope interaction changes the collimation of the outflow is thus unclear.

According to our model, the outflow opening angle is determined by the force balance between the wind and the envelope. With the wind strength increases or the envelope becomes less turbulent, the opening angle of the outflow becomes larger, and the outflow tends to decollimate. Our model provides a quantitative explanation of the de-collimation of the outflow in the context of the wind-envelope interaction.

Turbulence in the envelope plays a vital role in our model: the shape of the outflow cavity is determined by the hydrostatic balance between the wind and the envelope, and an increase in the strength of the wind can decrease the collimation of the outflow, while an increase of the turbulence in the envelope can increase the collimation of the outflow. Turbulence helps to collimate the outflow.

\subsection{Mass entrainment of the outflow layer}

As discussed in Sect. 1, the growth of the outflow depends on the way matter is entrained into it.
The key parameter that characterizes the mass entrainment process is the local mass entrainment rate, which is the amount of matter entrained per given time interval per surface area. The entrainment rate $\dot{m}$ can therefore be defined as

$\dot{m}=\frac{M_{\text {entrained }}}{\mathrm{d} s \times \mathrm{d} t}$,

where $M_{\text {entrained }}$ is the amount of gas entrained, ds represents the unit surface area and dt represents the time interval. The entrainment rate depend on the density and the velocity involved in the entrainment process. Therefore, the general form of the entrainment rate can be formulated as

$\dot{m}=f_{\text {entrainment }}\left(c_{\mathrm{s}}, \sigma_{\text {turb }}, v_{\text {shear }}\right) \times \rho_{\text {envelope }}$,

where $c_{\mathrm{s}}, \sigma_{\text {turb }}$ and $v_{\text {shear }}$ are the sound speed, turbulent speed and the velocity difference between the shearing layer and the envelope, and $\rho_{\text {envelope }}$ is the density of the envelope.

The factor $f_{\text {entrainment }}$ takes into account the effect of different speeds on the entrainment rate, and has the dimension of the velocity. In our case, the relative speed between the outflow and the envelope $v_{\text {shear }}$ is much higher than the velocity dispersion of the envelope $\sigma_{\text {turb }}$, and $\sigma_{\text {turb }}$ is higher than the sound speed $\left(v_{\text {shear }} \gg \sigma_{\text {turb }}>c_{s}\right)$.

The entrainment process is driven by the shearing motion and limited by the transport properties of the medium. The shearing motion is characterized by $v_{\text {shear }}$ and the transport process is characterized by $\sigma_{\text {turb }}$ and $c_{\mathrm{s}}$. As $v_{\text {shear }} \gg \sigma_{\text {turb }}$, the entrainment rate is limited by the transport and is determined by a combination of $\sigma_{\text {turb }}$ and $c_{\mathrm{s}}$. In a turbulent medium, turbulence dominates the transport. Therefore, in our model, $f_{\text {entrainment }}$ is determined by turbulence. We thus take

$f_{\text {entrainment }}=\alpha \sigma_{\text {turb }}$,

where $\alpha$ is a dimensionless factor that characterizes the efficiency of the entrainment process. We therefore parametrized the entrainment rate in the presence of turbulent motion as

$\dot{m}=\alpha \sigma_{\text {turb }} \rho_{\text {envelope }}$,

where $\sigma_{\text {turb }}$ is the characteristic velocity of turbulent motion in the medium. We assume $\alpha=0.1$ in this work.

Historically, turbulent mixing has long been proposed as a mean of entraining mass into the outflow (e.g. Canto \& Raga 1991; Watson et al. 2004). However, these models are doubted because the entrainment rate is far too small to account for the actual mass the outflow (Churchwell 1997). Realizing the importance of turbulence for enhancing the mass and momentum transport, we here propose that the argument by Churchwell (1997) no longer holds in our cases because turbulent mixing can be significantly enhanced when the ambient medium is turbulent.

\subsection{Mass and momentum conservation in the entrainment layer}

Based on the assumption that the outflow gas is the entrained envelope material, we formulate the equations that govern the evolution of the outflow. The geometry of the outflow is illustrated in Fig. 3. We defined the $x$-axis to be along the wall of the outflow cavity and $v_{x}$ as the average velocity of the outflow along the $x$-axis. By doing this, our $x$-axis is no longer a straight line. We used this construction for simplicity. $\omega$ is the same as illustrated in Fig. 1, which gives a circumference $l_{\mathrm{c}}$ of

$l_{\mathrm{c}}=2 \pi \omega$. 


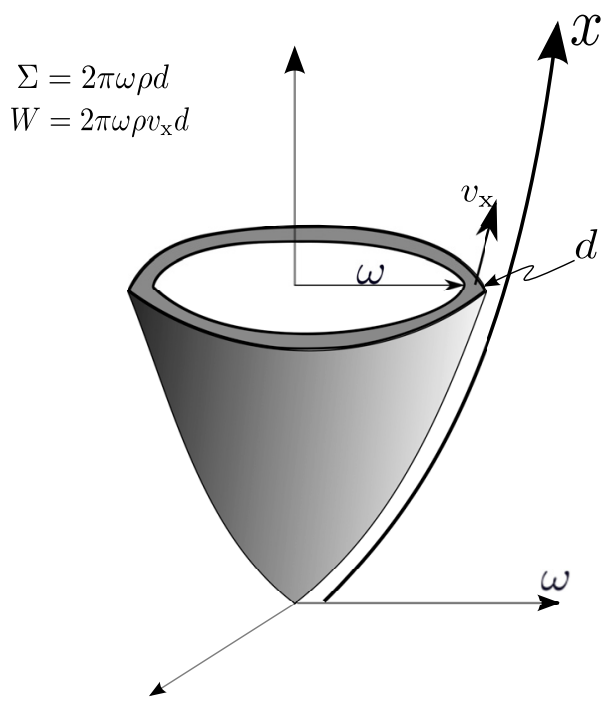

Fig. 3. Cartoon illustrating the geometry of the outflow layer and the definition of physical quantities in Sect. 2.4.

In Fig. 3, $\rho$ is the average density and $d$ is the geometrical thickness of the outflow layer. In our case, the outflow is geometrically thin $(d \ll l)$.

$\Sigma$ is the integrated density, which is defined as

$\Sigma=l_{\mathrm{c}} \rho=2 \pi \omega \rho d$,

and $W$ is the integrated momentum, which is defined as

$W=l_{\mathrm{c}} \rho v_{x}=2 \pi \omega \rho v_{x} d$,

where $v_{x}$ is the average outflow velocity along the outflow wall. Note that here $d$ is introduced for convenience. At a given position, we calculated $\Sigma$ and $W$ first. $d$ can then be evaluated as $d=\Sigma /(2 \pi \omega \rho)$.

The equations of motion of the outflow layer describe the conservation of the mass and the momentum,

$$
\begin{aligned}
\frac{\partial}{\partial t} \Sigma(x, t)+\frac{\partial}{\partial x}\left(v_{x}(x, t) \Sigma(x, t)\right) & =S_{\Sigma}(x) \\
\frac{\partial}{\partial t} W(x, t)+\frac{\partial}{\partial x}\left(v_{x}(x, t) W(x, t)\right) & =S_{\mathrm{W}}(x, \Sigma),
\end{aligned}
$$

where $W=v_{x} \Sigma$.

To solve it, we need the initial conditions $\Sigma(x, t=0)$ and $W(x, t=0)$.

We still need to specify the source terms $S_{\Sigma}$ and $S_{\mathrm{W}}$. According to the geometry in Fig. $1, S_{\Sigma}$ can be expressed as the sum of the mass flow from the wind and the mass flow from the envelope,

$S_{\Sigma}=S_{\Sigma \text { wind }}+S_{\text {¿envelope }}$

where

$S_{\text {¿wind }}=\rho_{\text {wind }} v_{\text {wind }} \sin \phi \times 2 \pi \omega \times \beta$

and according to Eq. (14),

$S_{\text {Eenvelope }}=\alpha \rho_{\text {envelope }} \sigma_{\text {turb }} \times 2 \pi \omega$.

Here, $S_{\Sigma \text { wind }} \ll S_{\Sigma \text { envelope }}$. Most of the mass in the outflow comes from the envelope.

$S_{\mathrm{W}}$ is the source term for the integrated momentum. $S_{\mathrm{W}}$ consists of the momentum injection by the wind, as well as the change of the momentum due to gravity. $S_{\mathrm{W}}$ can then be expressed as,

$$
\begin{aligned}
S_{\mathrm{W}} & =S_{\text {Wwind }}+S_{\text {Wgravity }} \\
& =\rho_{\text {wind }} v_{\text {wind }}^{2} \sin \phi \cos \phi \times 2 \pi \omega \times \beta+\Sigma g,
\end{aligned}
$$

where $g=G M_{*} / r^{2} \cos \phi$ is the acceleration due to gravity. Here, $M_{*}$ is the stellar mass $\left(10 M_{\odot}\right)$, and $\cos \phi$ comes from projecting the gravity onto the wall of the outflow cavity. $\beta$ is the wind efficiency. It characterizes the efficiency with which the mixing layer absorbs mass and momentum from the wind. We took 0.3 as its fiducial value.

\subsection{Local linear growth regime}

Here we make an order-of-magnitude analysis of Eqs. (18). The first terms are the transient terms $\frac{\partial}{\partial t} \times P$. The second terms are the advection terms, $\frac{\partial}{\partial x}\left(v_{x} \times P\right)$, and the third terms are the source terms $S_{P}$. Here, $P$ can be either $\Sigma$ or $W$. If the outflow has initial mass and momentum distribution $P_{0}=\Sigma_{0}$ or $W_{0}$, the mass/momentum accumulation timescales can be written as

$t_{\text {accumulation }}=P_{0} / S_{P}$.

and the advection timescales can be written as

$t_{\text {advection }}=L / v_{\mathrm{x}}$.

Here, $L$ is the physical scale of the outflow, which is about 1 parsec.

The mass/momentum accumulation timescale is dependent on the initial mass and momentum $\Sigma_{0}$ and $W_{0}$. If the majority of the mass and momentum of the outflow comes from the turbulence mixing process, $\Sigma_{0}$ and $W_{0}$ should be insignificant, therefore $t_{\text {accumulation }}$ is expected to be very short. Because we lack the knowledge on the initial mass and momentum distribution of the outflow, we assumed that the majority of the outflow mass and momentum comes from the turbulence mixing process. This assumption is valid as long as the outflow age is larger than the accumulation timescale $t_{\text {accumulation }}$ (Eq. (23)) is long. We studied the behaviour of Eqs. (18) by analysing the importance of different terms. For simplicity, we neglected the momentum added to the outflow due to self-gravity, therefore $W(x, \Sigma)$ in Eqs. (18) can be approximated as $W(x)$.

The advection timescale $t_{\text {advection }}$ is the time required for matter to travel throughout the outflow. If the outflow has an age of $t_{\text {outflow }}$, the transient term is

TransientTerm $=\frac{\partial P}{\partial t} \sim \frac{P}{t_{\text {outflow }}}$

and the advection term is

AdvectionTerm $=\frac{\partial v_{x} P}{\partial x} \sim \frac{v_{x} P}{L} \sim \frac{P}{t_{\text {advection }}}$.

If we take the ratio between the advection term and the transient term, we have

$\frac{\text { AdvectionTerm }}{\text { TransientTerm }} \sim \frac{v_{x} / L}{1 / t_{\text {outflow }}} \sim \frac{t_{\text {outflow }}}{t_{\text {advection }}}$,

where by definition $t_{\text {advection }}=L / v$. In our typical case, $t_{\text {outflow }}=$ $1 \times 10^{4} \mathrm{yr}$ and $t_{\text {advection }} \sim L / v_{x} \sim 1 \mathrm{pc} / 30 \mathrm{~km} \mathrm{~s}^{-1} \sim 1 \times 10^{5} \mathrm{yr}$. $t_{\text {outflow }}<t_{\text {advection, }}$, therefore advection is not important in the whole outflow. In this case, the advection effect can smooth out the density variations at the small scale, but can not alternate 
the structure of the whole outflow significantly. This is also true for the outflow from the class 0 low-mass protostars (Arce et al. 2007).

If the age of the outflow is small ( $\left.t_{\text {outflow }} \ll t_{\text {advection }}\right)$, as it is most cases, we can neglect the advection terms in Eqs. (18), therefore we have

$$
\begin{aligned}
\frac{\partial}{\partial t} \Sigma(x, t) & =S_{\Sigma}(x) \\
\frac{\partial}{\partial t} W(x, t) & =S_{\mathrm{W}}(x) .
\end{aligned}
$$

The surface density of the outflow can then be calculated as

$\Sigma(x, t)=S_{\Sigma}(x) \times t_{\text {outflow }}$,

and the velocity of the outflow can be calculated as

$v_{\mathrm{x}}(x, t)=\frac{S_{W}}{S_{\Sigma}}$.

In this case, the velocity profile of the outflow is fixed and the surface density of the outflow grows linearly with time.

\subsection{Advection-term-dominated regime}

For the aged low-mass outflows (e.g outflows with $t \sim 10^{7} \mathrm{yr}$ ), we have $t_{\text {outflow }} \sim t_{\text {advection, }}$, and the advection effect can alter the mass distribution of the outflow significantly. Neglecting the transient terms in Eqs. (18), we have

$$
\begin{aligned}
\frac{\partial}{\partial x}\left(v_{x}(x, t) \Sigma(x, t)\right) & =S_{\Sigma}(x) \\
\frac{\partial}{\partial x}\left(v_{x}(x, t) W(x, t)\right) & =S_{\mathrm{W}}(x),
\end{aligned}
$$

hence

$$
\begin{aligned}
& v_{x}=\frac{\int S_{W} \mathrm{~d} x}{\int S_{\Sigma} \mathrm{d} x} \\
& \Sigma=\frac{\left(\int S_{\Sigma} \mathrm{d} x\right)^{2}}{\int S_{\mathrm{W}} \mathrm{d} x} .
\end{aligned}
$$

Here, both the velocity and the surface density exhibit stationary profiles. By comparing Eqs. (30) and (31) with Eq. (34), we find that when the outflow age is high, the advection term dominates the transient term, which prevents the outflow surface density from growing to infinity.

\subsection{Role of gravity}

Here we consider the role of the gravity term in the structure of the outflow. According to Eq. (22), the contribution of gravity is quantified by the ratio

$$
f_{\text {gravity }}=\frac{\Sigma g}{\rho_{\text {wind }} v_{\text {wind }}^{2} \times 2 \pi \omega \times \beta} \sim \frac{\rho d 2 \pi \omega g \alpha}{\rho_{\text {wind }} \times v_{\text {wind }}^{2} \times 2 \pi \omega \beta},
$$

where $d=\sigma t \alpha$ is used. Here, $d$ is the thickness of the outflow, $\alpha$ is the entrainment efficiency, $\sigma$ is the velocity dispersion of the envelope and $t$ is the outflow age. From this we have

$f_{\text {gravity }}=\frac{g t}{\sigma} \times \frac{\alpha}{\beta}$.

Here, $\sigma$ is the velocity dispersion of the envelope, $t$ is the age of the outflow, and $g \sim G M_{*} / r^{2}$ is the local gravity. Since $g \sim r^{-2}$, gravity will be important at regions close to the protostar, and it will be important when the mass of the outflow has grown significantly.

Considering the inner region of the outflow, we have $M_{*}=$ $10 M_{\odot}, r=0.1 \mathrm{pc}$ and $t=10^{4} \mathrm{yr}$. Therefore, $f_{\text {gravity }} \sim 10^{-2}$ and the gravity from the protostar is negligible. However, at regions closer to the protostar, gravity must play an important role in slowing down the outflow.

\subsection{Numerical results}

To illustrate how the mass and the momentum of the outflow grows with time, we assume a static envelope and a wind with a constant mass-loss rate and followed the evolution of the entrainment layer by solving Eqs. (18)-(22) numerically. The cavity shapes are calculated in Sect. 2.2. At $x=0$, we imposed

$\frac{\partial \Sigma}{\partial x}=0$

$\frac{\partial W}{\partial x}=0$

and set the initial condition so that

$\Sigma(x)=S_{\Sigma} \Delta t_{0}$

$v_{x}(x)=1 \times 10^{-4} \mathrm{~cm} \mathrm{~s}^{-1}$,

where $\Delta t_{0}=100 \mathrm{yr}$. The equations are solved using FiPy (J. E. Guyer, D. Wheeler \& J. A. Warren) ${ }^{1}$.

We are mainly interested in the transient-term-dominated regime, since because this regime is relevant to all high-mass protostellar outflows and the majority of the young low-mass protostellar outflows.

In Fig. 4, we plot the results from our calculations for a realistic $10^{4}$ yr outflow and unrealistically old $10^{7}$ yr outflow. In our $10^{4}$ yr outflow, the numerical solution agrees quite well with the analytical solutions (Sect. 2.5). At small radius, the numerical solutions show lower velocity. This is because the matter in regions close to the protostar is slowed down by the gravity from the protostar.

We also plot the results of an outflow with an unrealistically long lifetime of $10^{7} \mathrm{yr}$. Because here gravity plays a much more significant role in slowing down the outflow (Sect. 2.7), the velocity of the outflow at the central part of the outflow becomes much lower.

In most cases, the effect of gravity is negligible in large portions of the outflow, the analytical solutions can be taken as good approximations to the structure of the outflow. However, it is difficult for the outflow to enter the advection-dominated regime, since here gravity tends to dominate the velocity structure of the outflow and to slow down the outward motion before advection is able to change the structure of the whole outflow significantly.

The final state we obtained from solving the equations of mass and momentum conservation in the local linear growth regime is representative of the real structure of the outflow, as long as the mass and momentum added to the outflow is much larger than the initial mass and momentum of the outflow. If the strength of the wind or the structure of the envelope changed during the evolution of the system, the final state of the outflow will change accordingly. Therefore, the system will evolve towards a new state, and the time for this evolution to take place is again the accumulation timescale (Eq. (23)). The older the outflow becomes, the more difficult it is for the outflow to relax to the new state.

1 Avaliable at http://www.ctcms.nist.gov/fipy/ 
Guang-Xing Li et al.: Turbulent outflow mode
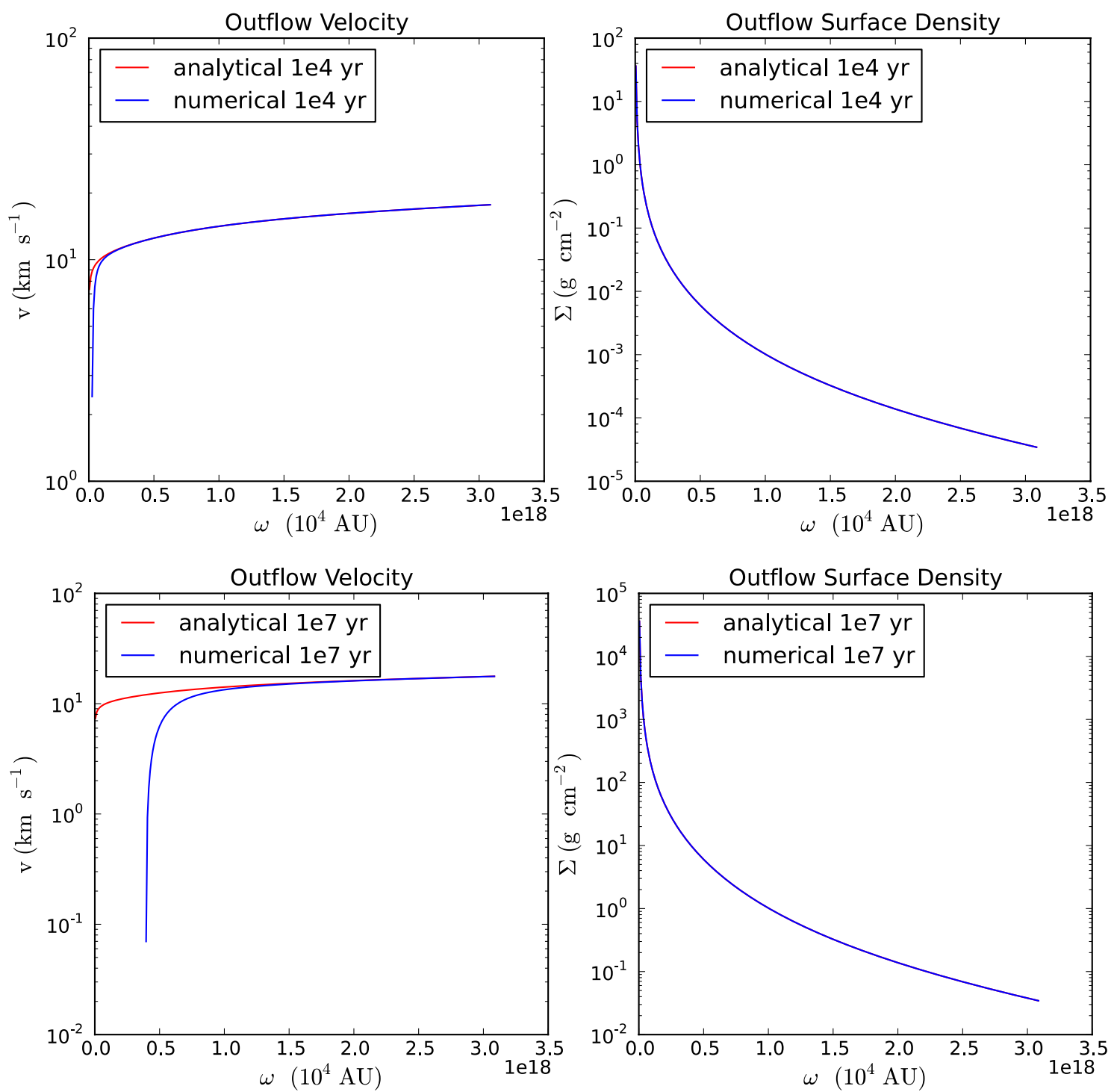

Fig. 4. Velocity and surface density distribution of the outflow calculated by solving Eqs. (18). The blue lines are the numerical results and the red lines are results calculated by assuming the local conservation of energy and momentum (Sect. 2.5). The upper panels are the results for a $10^{4}$ yr outflow. The lower panels are the results for an unrealistically old $\left(10^{7} \mathrm{yr}\right)$ outflow. At smaller radii, the deviation of the numerical solutions from the analytical solutions are caused by the gravity term in Eq. (22).

In our present work, we assumed that the wind and envelope remain stationary. Our solution is valid when the structure of the wind and the structure of the envelope change slowly. Since detailed observations of the way in which the wind and envelope evolve are currently unavailable, we assume this simplicity. If the wind and the envelope both evolve significantly, it is still possible to calculate the structure of the outflow by making some modifications to the framework presented.

\subsection{Structure of the entrainment layer}

To fully specify the structure of the outflow, we need to know the structure of the entrainment layer. The gas motion inside the entrainment layer is dominated by turbulence, and its velocity can be expressed as the sum of a mean component and a fluctuating component (Reynolds 1895). There are many experiments and numerical simulations that study the structure of such a mixing layer (e.g. Champagne et al. 1976; Bell \& Mehta 1990; Rogers \& Moser 1994), from which we obtained the structures of the mean velocity and the fluctuating (random) velocity, and parametrized them.

We found that the structure of the entrainment layer is universal and can be represented in a simple parametrized way. We fitted the mean density, the mean velocity and the turbulent velocity structures of Rogers \& Moser (1994), and applied the fitting formula to our case.

The fitted density and mean velocity distributions take the forms of

$\rho(\xi)=\frac{-\operatorname{erf}(4 \xi-2)+1}{2} \rho_{\text {envelope }}$,

and

$v(\xi)=\frac{\operatorname{erf}(4 \xi-2)+1}{2} v_{\text {norm }}$,

where erf is the error function, and $\xi$ is defined as

$\xi=\frac{x}{2 d}$. 


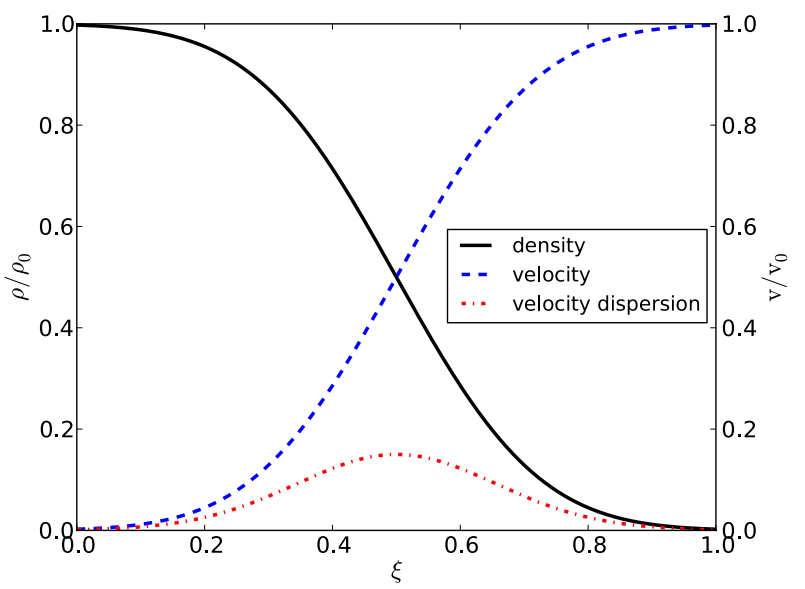

Fig. 5. Density and velocity structure of the entrainment layer. The (black) solid line denotes the density structure (Eq. (41)), the (blue) dashed line denotes the velocity structure (Eq. (42)), and the (red) dotted line denotes the structure of the fluctuating velocity (Eq. (44)). $\xi$ is defined in Eq. (43).

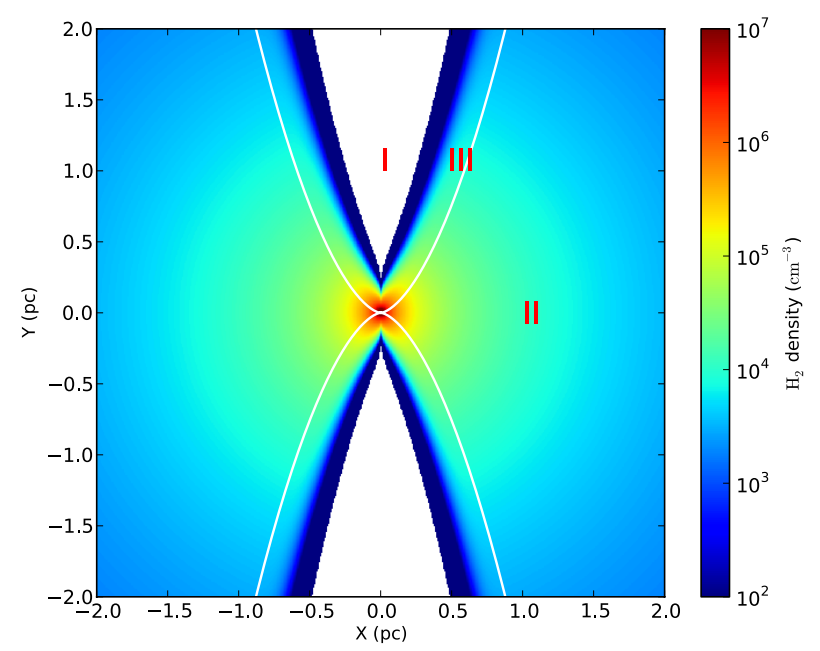

Fig. 6. Density structure of the outflow. Region I represents the inner cavity blown by the wind, region II represents the envelope, and region III represents the outflow layer. The wind mass-loss rate is $1.5 \times 10^{-3} M_{\odot} \mathrm{yr}^{-1}$. The thickness of the entrainment layer has been scaled up by a factor of 30 for clarity.

$d$ is defined as $d=\Sigma / \rho_{\text {envelope }}$, and $v_{\text {norm }}=5 \times v_{x}$ due to the normalization requirement.

The distribution of the fluctuating velocity of the entrainment layer takes the form

$$
\begin{aligned}
v_{\text {turb }} & =v_{\text {turb_norm }}(\operatorname{erf}(4 \xi-2)+1)(-\operatorname{erf}(4 \xi-2)+1) \\
& =v_{\text {turb_norm }}\left(1-\operatorname{erf}^{2}(4 \xi-2)\right),
\end{aligned}
$$

where $v_{\text {turb_norm }}=0.15 \times v_{\text {norm }}$. Figure 5 shows the structure of density, mean velocity, and fluctuating velocity inside the entrainment layer.

Figure 6 shows the density distribution of our model, from which the entrainment layer can be identified as the region between the outflow cavity and the envelope.

\section{Observational tests}

Observationally, molecular outflows are frequently mapped in the emission from rotational transitions of molecules such as $\mathrm{CO}$ and $\mathrm{HCO}^{+}$. These mapping observations can measure the intensity of the line emission from the outflow in the form of threedimensional $x y v$ ( $x$-position- $y$-position-velocity) data cubes. $\mathrm{CO}$ is the most abundant and widespread molecule in the interstellar medium apart from the difficult-to-observe $\mathrm{H}_{2}$, and has been used to trace the bulk of molecular gas. CO observations (Lada 1985; Arce et al. 2007, and references therein) have revealed a diversity of molecular outflows, some of which show remarkably regular morphologies (e.g. Qiu et al. 2009).

Here we concentrated on modelling the emission from our outflows and studied the connection between the physical structure of the outflow models and the structure of our outflow models observed in a 3D data cube (e.g. Cabrit \& Bertout 1986, 1990, 1992; Stahler 1994). To model the emission from the outflow, we need to know its dynamics and its abundance/excitation conditions. The dynamical structure of the outflow has been obtained in Sect. 2. However, the abundance and excitation are still uncertain: in regions close to the central protostar, the $\mathrm{CO}$ molecule can be photodissociated and cannot trace the gas anymore. The temperature of the outflow layer is determined by the balance between various heating and cooling processes, which are also uncertain. While these effects will influence the spacial and velocity distribution of the observed $\mathrm{CO}$ emission, the morphology of the outflow as well as its overall structure in the positionposition and position-velocity spaces are relatively unchanged. Therefore we assume that the $\mathrm{CO}$ abundance is $10^{-4}$ relatively $\mathrm{H}_{2}$ and that the outflow entrainment layer has a constant kinematic temperature of $\sim 100 \mathrm{~K}$. In the following discussions, we focus on the overall morphology of the outflow in the positionposition and position-velocity spaces.

For the velocity dispersion, velocity gradient and kinematic temperature from our model, we used a python version of RADEX (van der Tak et al. 2007) to calculate the population distribution of the $\mathrm{CO}$ molecule. We then used a ray-tracing code to calculate the line emission from our outflows. Our raytracing calculations were made with the help of LIME (Brinch \& Hogerheijde 2010).

Figure 7 shows a comparison between our model and the observations by Qiu et al. (2009). We show the integrated image of the outflow and the position-velocity diagram of the outflow cut along the major axis. The integrated images of the outflow show a regular conical shape in both the observations and our modellings, and the position-velocity diagram of the outflows from observations and modellings show similar shapes. In regions close to the protostar, the observations show a velocity dispersion of about $20 \mathrm{~km} \mathrm{~s}^{-1}$. This velocity dispersion comes from a continuous distribution of emission and is consistent with observational studies (e.g. Qiu et al. 2011; Cyganowski et al. 2011).

We propose that this feature is one strong evidence for the existence of the entrainment layer. By looking at one position in the image, we integrate through the whole entrainment layer. In one line of sight, this produces a continuous distribution of fluids that move at different speeds (Fig. 5). Seen from the spectral line profile, we can always identify a broad component, since the line emission traces the distribution of the mass, and the mass distribution inside the entrainment layer is continuous.

On the other hand, the observation shown in Fig. 7 is difficult to understand in the context of the wind-driven shell model. In that model, the velocity of the outflow is proportional to the distance from the protostar and should vanish at close vicinity of the protostar, which is not observed (Fig. 7). Moreover, the outflow speed are high at regions far away from the protostar because only a high expansion velocity can make the gas move 
Guang-Xing Li et al.: Turbulent outflow mode

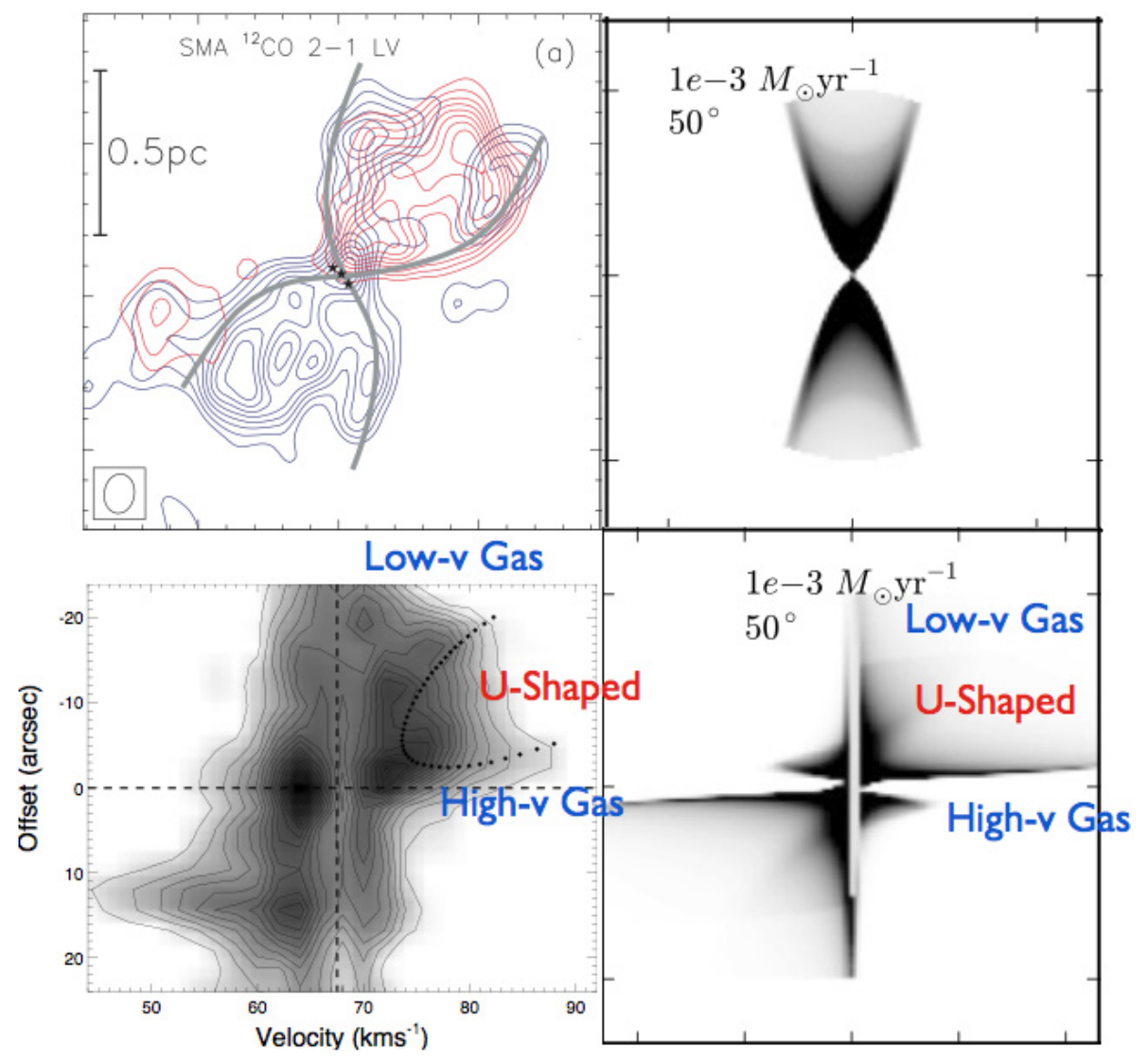

Fig. 7. Comparison between the observations by Qiu et al. (2009) and the predictions from our wind-driven turbulent entrainment model. The upper left panel shows the morphology of the outflow observed by Qiu et al. (2009), the lower left panel shows the position-velocity structure of the observed outflow. The upper right panel shows the morphology of the outflow from our modelling, the lower middle panel shows the position-velocity structure of our outflow model cut along its major axis. The outflow model has a mass-loss rate of $1.0 \times$ $10^{-3} M_{\odot} \mathrm{yr}^{-1}$ and an inclination angle of $50^{\circ}$. For both the observations (lower left panel) and our modelling (lower right panel), the $\mathrm{U}$-shaped region is the region in the positionvelocity diagram where the structure of the outflow exhibits a U shape, the Low- $v$ gas region is the region where the gas has a relative small velocity at regions far from the protostar, and the High- $v$ gas region is the region where the gas has a relatively high velocity in the close vicinity to the protostar.

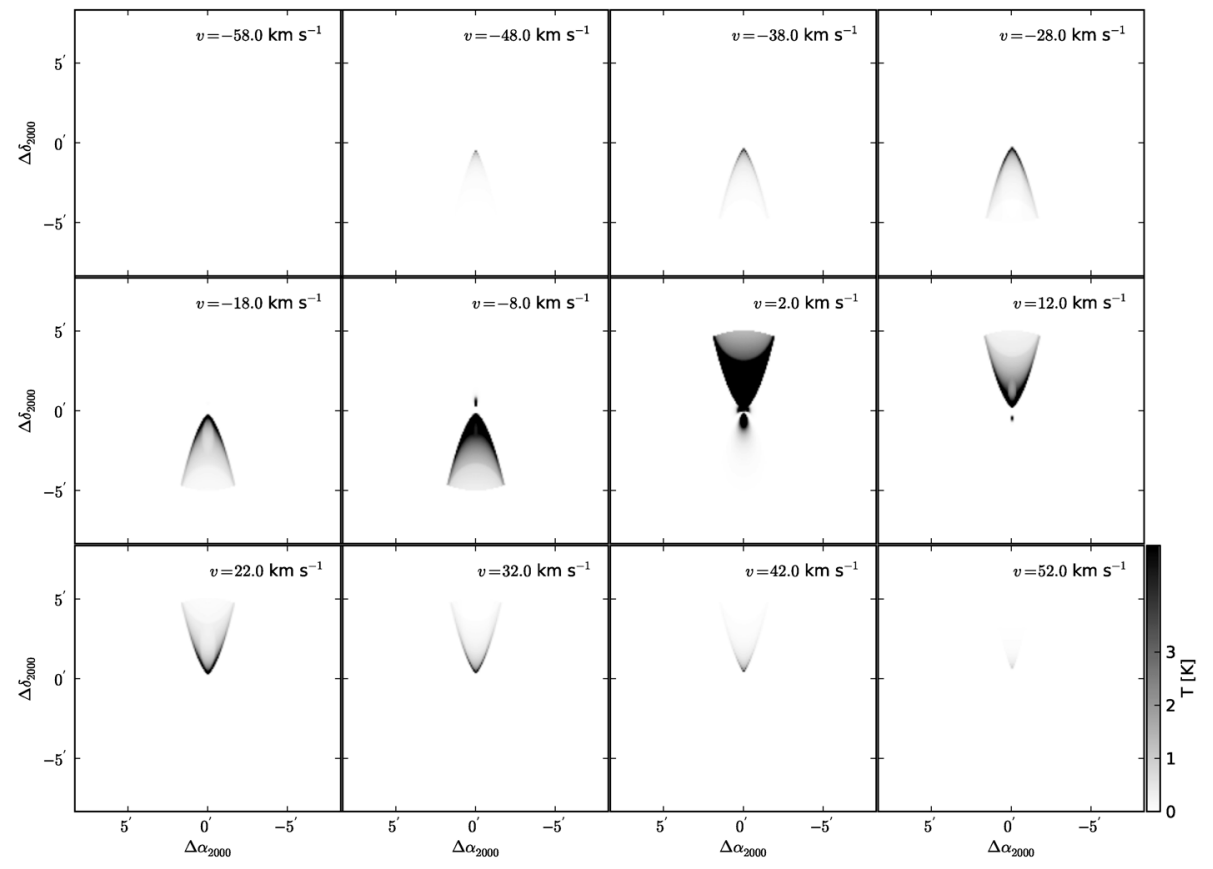

Fig. 8. $\mathrm{CO}(3-2)$ channel map of an outflow that has a mass-loss rate of $1.0 \times 10^{-3} M_{\odot} \mathrm{yr}^{-1}$ and an inclination angle of $50^{\circ}$. far. But in the observations we can still see low-velocity gas in regions far from the protostar.

Figure 8 shows a channel map of an outflow with a mass-loss rate of $1.0 \times 10^{-3} M_{\odot} \mathrm{yr}^{-1}$ and an inclination angle of $50^{\circ}$. The inner part of the outflow is visible in most channels, implying a broad velocity spread at this location. This velocity spread is a direct consequence of the turbulent entrainment process.
Figures 9 and 10 show the calculated images and positionvelocity diagrams of outflows from protostars with different mass-loss rates viewed at different inclination angles. The massloss rate takes values of $0.5,1,1.5$, and $\times 10^{-3} M_{\odot} \mathrm{yr}^{-1}$, the inclination angles values of $10^{\circ}, 30^{\circ}$, and $50^{\circ}$. The calculated outflows exhibit a variety of morphologies. They also share some common characteristics: the outflow gas can reach a high velocity in regions close to a protostar; in regions far away from the 


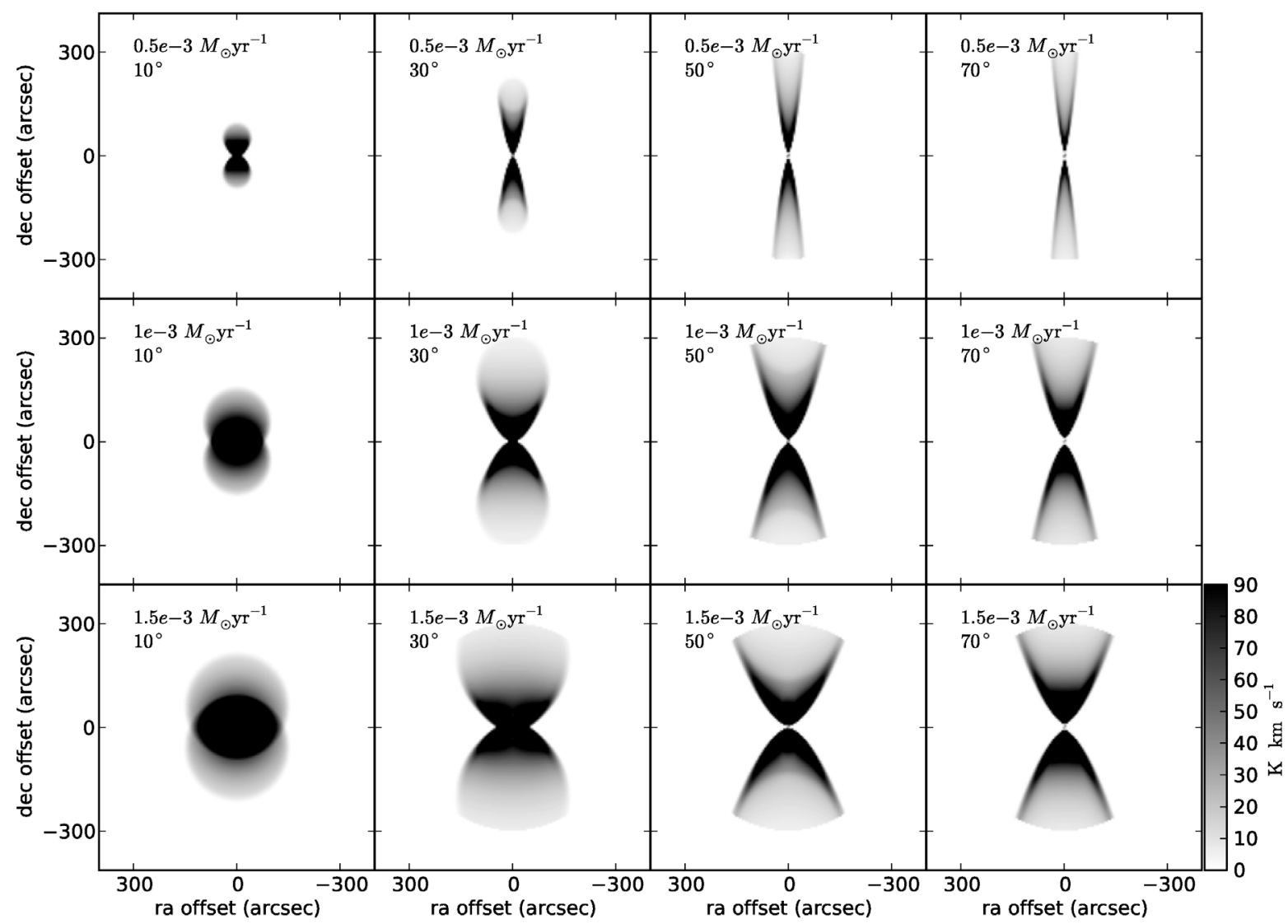

Fig. 9. Velocity-integrated $\mathrm{CO}(3-2)$ images of outflows from our model with different wind mass-loss rates and inclination angles.

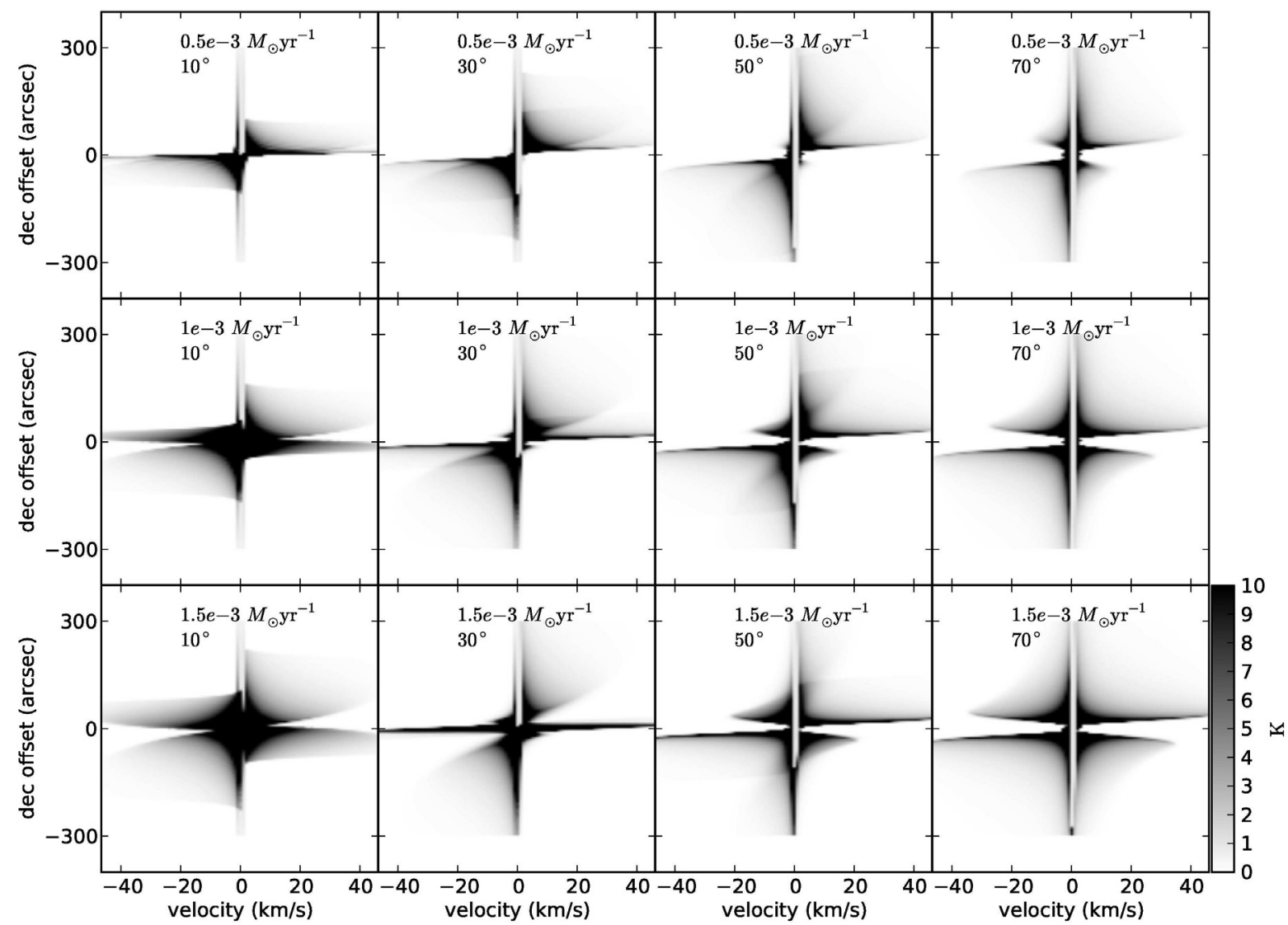

Fig. 10. $\mathrm{CO}(3-2)$ position-velocity diagram cut along the major axis of our outflow models with diferent wind mass loss rates and inclination angles. 
protostar, there is still gas with low velocities. These features are natural outcomes of the entrainment process (Sect. 2.9) and can be observed under different circumstances.

\section{Outflow entrainment as a universal process}

Outflows are ubiquitously observed in a variety of situations, which include the formation of low-mass stars and high-mass stars, and the situation in which a wind blown by an AGN interacts and entrains the galactic-scale ambient gas (Alatalo et al. 2011; Tsai et al. 2012). Here we considered the possibility that these outflows have a common origin, in that all these outflows are formed through the interaction between the wind and the ambient gas in the form of turbulent entrainment.

In Sect. 4.1, using the turbulent core model of massive star formation (McKee \& Tan 2002, 2003), we show that outflows from low and high-mass protostars can consistently be interpreted as resulting from an universal entrainment process. In Sect. 4.2, we derive universal scaling relations that can be used to estimate the mass and velocity of the outflows, and suggest that the AGN-driven outflows can be consistently explained in our model. In Sect. 4.3, using the insights obtained from the scaling analysis, we predict the existence of dwarf outflows in clusterforming regions, and in Sect. 4.4 we discuss the self-similarity of our model.

\subsection{Universal picture of protostellar outflows}

As suggested by McKee \& Tan (2002, 2003), the formation of high-mass stars can be viewed as a scaled-up version of the standard low-mass star formation theory from gas cores, where most of the pressure support is due to a combination of turbulence and magnetic fields instead of thermal pressure. In this picture, a high level of turbulence prevails in the high-mass star forming regions, causing significant turbulent ram-pressure of the ambient medium. The timescale for the formation of high-mass stars is short, and the accretion rate onto the high-mass protostar is much higher than in the low-mass case.

Our model of a turbulent entrainment outflow is self-similar in nature. By combining it with the self-similar star formation model, we can obtain a universal description of outflows from low and high-mass protostars.

Considering the fiducial case in McKee \& Tan (2003), the accretion rate onto the protostar can be estimated as

$\dot{m}_{*} \sim 4.6 \times 10^{-4} \times\left(\frac{m_{* f}}{30 M_{\odot}}\right)^{3 / 4} \times \Sigma_{\mathrm{cl}} \times\left(\frac{m_{*}}{m_{* f}}\right)^{0.5} M_{\odot} \mathrm{yr}^{-1}$,

where $m_{*}$ is the current mass of the protostar, $m_{* f}$ is the final mass of the protostar when the accretion has been finished, and $\Sigma_{\mathrm{cl}}$ is the surface density of the clump. High-mass star-forming regions are characterized by a high value of $\Sigma_{\mathrm{cl}}$ (McKee \& Tan 2003). The pressure of the wind from the protostar can be estimated as

$$
\begin{aligned}
\frac{p_{\text {wind }}}{k_{\mathrm{B}}}= & \frac{\dot{m}_{\text {wind }} \times v_{\text {wind }}}{\pi r^{2}} \\
= & 0.77 \times 10^{8} \times\left(\frac{m_{* f}}{30 M_{\odot}}\right)^{3 / 4} \times \Sigma_{\mathrm{cl}}^{3 / 4} \\
& \times\left(\frac{r}{\mathrm{pc}}\right)^{-2} \times\left(\frac{m_{*}}{m_{* f}}\right)^{0.5} \mathrm{~K} \mathrm{~cm}^{-3},
\end{aligned}
$$

where $k_{\mathrm{B}}$ is the Boltzmann constant, and we assumed $\dot{m}_{\text {wind }}=$ $1 / 3 \times \dot{m}_{*}$ (Najita \& Shu 1994, where $\dot{m}_{*}$ is the total accretion rate onto the protostar) and $v_{\text {wind }}=600 \mathrm{~km} \mathrm{~s}^{-1}$. The average pressure of the clump is expressed as (McKee \& Tan 2003)

$$
\frac{\bar{p}_{\mathrm{cl}}}{k_{\mathrm{B}}}=\frac{0.88 G}{k_{\mathrm{B}}} \Sigma_{\mathrm{cl}}^{2}=4.2 \times 10^{8} \Sigma_{\mathrm{cl}}^{2} \mathrm{~K} \mathrm{~cm}^{-3} \text {. }
$$

For typical parameters, the average pressure of the envelope $\bar{p}_{\mathrm{cl}}$ is similar to the average pressure of the wind at $1 \mathrm{pc}$, which is approximately the physical size of the outflow. This justifies our suggestion that the ram-pressure of the wind and the rampressure of the ambient medium are similar.

The collimation of the outflow is determined by the ratio between the turbulent ram-pressure of the envelope and the rampressure of the wind. Stronger winds lead to less-collimated outflows while more turbulent envelopes lead to highly collimated outflows. To summarize these effects, we define the dimensionless collimation parameter $\eta$ of the outflow:

$$
\begin{aligned}
\eta & =\frac{\bar{p}_{\mathrm{cl}}}{p_{\text {wind }}} \\
& =5.5 \times\left(\frac{r}{\mathrm{pc}}\right)^{2}\left(\frac{m_{*}}{m_{* f}}\right)^{-1 / 2} \Sigma_{\mathrm{cl}}^{1 / 2}\left(\frac{m_{* f}}{30 M_{\odot}}\right)^{-3 / 4},
\end{aligned}
$$

with larger $\eta$ implying more collimated outflows.

Equation (48) has several implications.

The ratio $\eta$ is proportional to $r^{-2}$, which means that the pressure of the envelope gradually dominates the pressure of the outflow tends to collimate the outflow with increasing distance from the protostar. This may explain why many outflows exhibit a recollimated shape, that is, the opening angle of the outflow becomes smaller as we moves away from the protostar (e.g. L1157, Bachiller \& Perez Gutierrez 1997).

Also, $\eta \sim \Sigma_{\mathrm{cl}}^{1 / 2}$, which means that higher external pressure (stronger turbulence) leads to more collimated outflows. This agrees with the results in Sect. 2.2, and indicates that turbulence can collimate the outflow.

Third, if the pressure of the clump $\Sigma_{\mathrm{cl}}$ is roughly constant, the more massive the star is, the less collimated is the outflow. This is because $\eta$ depends on the final mass of the protostar, $\eta \sim m_{* f}^{-3 / 4}$. The more massive the star, the stronger the wind it has. This stronger wind will push the envelope and leads to a less collimated outflow. If several stars form in a clustered way (Qiu et al. 2011), protostars of different mass will share one common environment. We then expect that more massive protostars produce less-collimated outflows.

\subsection{Outflow mass and velocity}

According to our model, the mass of the outflow is determined by the efficiency with which the outflow entrains the envelope gas. Given the opening angle, the mass of the outflow can be estimated as

$M_{\text {outflow }} \sim \alpha \rho_{\text {average }} \sigma_{\text {average }} \times t \times L^{2}$,

where $L$ is the physical scale of the outflow and $\rho_{\text {average }}$ and $\sigma_{\text {average }}$ are the average density and velocity dispersion, respectively. The average pressure of the envelope is

$p_{\text {envelope }} \sim \rho_{\text {average }} \times \sigma_{\text {average }}^{2}$.

The momentum of the outflow is determined by the amount of momentum injected by the wind and can be estimated as

$P_{\text {outflow }} \sim \dot{M}_{\text {wind }} \times t \times v_{\text {wind }} \times \beta$, 
and the ram-pressure of such a wind can be estimated as

$p_{\text {wind }} \sim \frac{\dot{M}_{\text {wind }} \times v_{\text {wind }}}{L^{2}}$.

If the cavity blown by the outflow is stable, the ram-pressure of the wind $p_{\text {wind }}$ is expected to be similar to the turbulent rampressure of the envelope, $p_{\text {envelope, }}$, and we have (from Eqs. (50) and (52))

$\dot{M}_{\text {wind }} \times v \sim \rho_{\text {wind }} \sigma_{\text {wind }} L^{2}$.

The outflow velocity is then (Eqs. (49), (50), and (53))

$$
\begin{aligned}
v_{\text {outflow }} & \sim \frac{P_{\text {outflow }}}{M_{\text {outflow }}} \sim \frac{\dot{M}_{\text {wind }} v_{\text {wind }}}{\rho_{0} \sigma_{0}} \times \frac{\beta}{\alpha} \\
& \sim \sigma_{\text {average }} \frac{\beta}{\alpha} \sim 3 \times \sigma_{\text {average }} .
\end{aligned}
$$

In the last step we inserted the numerical values of $\alpha$ and $\beta$. The velocity of the outflow is several times higher than the velocity dispersion of the envelope.

This fact can be understood as follows: If the wind and the envelope can establish hydrostatic equilibrium, then increasing turbulent velocity $\sigma$, the pressure of the wind has to scale according to $\sigma^{2}$ to balance wind pressure. Therefore, the momentum injection of the wind scales as $\sigma^{2}$. On the other hand, the mass supply of the outflow from the envelope in the form of the entrainment process only scales as $\sigma^{1}$. The outflow velocity, which is estimated as $P / M$, scales as $\sigma^{1}$.

These results outline one important property of the turbulent entrainment outflow, namely that the velocity of the outflow is higher than while still similar to the velocity dispersion of the ambient gas. This is independent of other parameters such as the strength of the wind, and is based only on the assumption that the wind and the envelope can establish hydrostatic equilibrium.

In recent Herschel-HIFI observations of high- $J$ CO emission in low and high-mass star-forming regions (San Jose-Garcia et al. 2013), it was found that the velocity of the outflows (traced by the FWHM of the broad ${ }^{12} \mathrm{CO}(10-9)$ line emission) is higher than while still similar to the velocity of the envelope (traced by the FWHM of the $\mathrm{C}^{18} \mathrm{O}(9-8)$ line). Also interesting is the fact that broader envelope line width is usually associated with broader outflow line width. This implies that the outflows from different regions share some physical similarities, and one possibility is that all these outflows come from the same entrainment process.

In a recently discovered AGN-driven outflow (Alatalo et al. 2011), the line width of the ambient gas (single-peaked central velocity component) is $\sigma_{\text {envelope }} \sim 100 \mathrm{~km} \mathrm{~s}^{-1}$, while the width of the broad line wing caused by the outflow activity is $v_{\text {outflow }} \sim 300 \mathrm{~km} \mathrm{~s}^{-1}$. Although velocity of the ambient gas and the velocity of the outflow are much higher than those of protostellar outflows, their ratio $v_{\text {outflow }} / \sigma_{\text {envelope }}$ is about 3 . This is similar to the ratio predicted in our model (Eq. (54)), which suggests that these outflows may be the outcome of the turbulent entrainment process working between the wind emitted by the central AGN and the galactic-scale ambient gas.

\subsection{Dwarf outflows}

As we have shown in Sect. 4.1, the emergence of an outflow depends on the pressure of the envelope and the pressure of the wind reaching a balance. This is not always true. If the wind from the protostars is not strong enough to blow away the envelope, we expect to see that the outflows have small spatial extent and are confined to small regions.

Such "dwarf" outflows may exist under several conditions. If several protostars form in a clustered way (e.g. Qiu et al. 2011), then the outflows from the low-mass protostars of different mass may be confined by the turbulence and appear as "dwarf outflow". Alternatively, such "dwarf" outflows may exist at regions where the wind from the protostar is extremely weak (e.g. VeLLOs, Lee et al. 2009; Dunham et al. 2010). Studies of such "dwarf" outflows will help to gain insights into the interaction between the wind and the envelope in extreme cases.

\subsection{Self-similarity of the model}

We explored the self-similar properties of our model. Each model is characterized by several parameters: a wind mass-loss rate $\dot{M}_{0}$, a turbulence velocity $\sigma_{0}$, a wind velocity $v_{0}^{\text {wind }}$, a density $\rho_{0}$, an outflow age $t_{0}$, and the entrainment parameters $\alpha_{0}$ and $\beta_{0}$. Our model is self-similar if when gravity is neglected. Here we derive the condition at which the structure of another outflow characterized by a different set of parameters $\left(\dot{M}_{1}, \sigma_{1}\right.$, $v_{1}^{\text {wind }}, \rho_{1}, t_{1}, \alpha_{1}$, and $\left.\beta_{1}\right)$ can be obtained from scaling of a different outflow.

The opening angle of the outflow is determined by the relative strength of the ram-pressure of the wind and the turbulent ram-pressure of the envelope. For it to be unchanged, we require

$\frac{\rho_{1} \sigma_{1}^{2}}{\rho_{0} \sigma_{0}^{2}}=\frac{\dot{M}_{1} \times v_{1}^{\text {wind }}}{\dot{M}_{0} \times v_{0}^{\text {wind }}}$.

If Eq. (55) is satisfied, the two outflows will have the same shape. But the total amount of gas contained in the outflows and their velocity are different. The amount of gas is proportional to the entrainment rate and proportional to the outflow age, therefore

$\frac{M_{1}}{M_{0}}=\frac{\rho_{1} \sigma_{1} t_{1} \alpha_{1}}{\rho_{0} \sigma_{0} t_{0} \alpha_{0}}$

As discussed in Sect. 4.2, the velocity of the outflow is proportional to the velocity dispersion of the envelope,

$\frac{v_{1}}{v_{0}}=\frac{\sigma_{1}}{\sigma_{0}}$.

These scaling relations help us to put outflows with different masses and velocities into a common picture.

\section{Conclusions}

We studied the interaction between the wind from a protostar and the ambient gas in the form of turbulent mixing and proposed a wind-driven turbulent entrainment model for protostellar outflows. In the model, the wind from a protostar is in hydrostatic balance with the gas in the turbulent envelope, and the outflowing gas is completely contained in the turbulent entrainment layer that develops between the wind and the envelope. Turbulence in the ambient gas plays two roles in our model: first, turbulent motion determines the shape of the outflow (Sect. 2.2). Second, turbulence contributes to the mass growth of the mixing layer (Sect. 2.4). Our model is a universal one in that it can explain the outflows from both low and high-mass protostars (Sect. 4). 
Our model can reproduce the geometry and kinematic structure of the observed outflows (Sect. 3, Figs. 7, 9 and 10). The main results from this study can be summarized as follows:

1. At the physical scale of an outflow, the average ram-pressure of wind is similar to the average ram-pressure of its envelope (Sect. 2.2, 4.1).

2. The opening angle of the outflow is dependent on the pressure balance between the wind and the envelope, therefore it can evolve if the pressure of the wind or the pressure of the envelope changes (Sects. 2.2, 4.1).

3. The ram-pressure of the wind tends to decollimate the outflow, the ram-pressure of the environment tends to collimate the outflow (Sects. 2.2, 4.1).

4. At one given point in the observed image of the outflow, the emission has a wide spread in velocity (Sects. 3, Fig. 10). Both high-velocity gas in the close vicinity of the protostar and low-velocity gas in regions far from the protostar exist as the results of the turbulent entrainment process.

5. If the outflow is formed through the turbulent entrainment process outlined here, the velocity spread of the outflow is expected to be about times the velocity spread of the ambient gas (Sect. 4.2). This is independent of the other physical parameters, such as the strength of the wind and the density of the region.

6. In clustered star-forming regions, we expect a population of dwarf outflows that are confined by the pressure of their environment into small regions (Sect. 4.3).

The universality of the entrainment process motivates speculation that the same entrainment process may be at work in AGNdriven outflows (Alatalo et al. 2011; Tsai et al. 2012). We suggest that the turbulent entrainment process works ubiquitously in nature and plays an important role in shaping outflows in many different situations.

Acknowledgements. We thank the anonymous referee for his/her insightful comments, which helped us to improve our draft. Guang-Xing Li is supported for this research through a stipend from the International Max Planck Research School (IMPRS) for Astronomy and Astrophysics at the Universities of Bonn and Cologne.

\section{Appendix A: Effect of centrifugal forces}

As the outflowing gas moves along the cavity, it exerts a centrifugal force on its surrounding. This effect has been considered in several previous works (e.g., Canto 1980; Canto \& Rodriguez 1980; Biro et al. 1993). The pressure produced by the centrifugal force can be estimated as

$p_{\text {centrifugal }} \sim v_{\text {outflow }}^{2} \kappa \rho_{\text {outflow }} d$,

where $v_{\text {outflow }}$ is the velocity of the outflow, $\kappa$ is the curvature of the outflow cavity, $\rho_{\text {outflow }}$ is the density of the outflow, and $d$ is the thickness of the outflow.

The ram-pressure of the envelope is

$p_{\text {envelope }} \sim \rho_{\text {envelope }} \times \sigma_{\text {turb }}^{2}$.

In our entrainment model, the density of the outflow is similar to the density of the envelope $\rho_{\text {outflow }} \sim \rho_{\text {envelope }}$, and the velocity of the outflow is several times the velocity dispersion of the envelope (Eq. (54)). Therefore, the ratio between centrifugal pressure and the pressure of the envelope is

$f=\frac{p_{\text {centrifugal }}}{p_{\text {envelope }}} \sim \frac{\rho_{\text {outflow }} \kappa d v_{\text {outflow }}^{2}}{\rho_{\text {envelope }} \sigma_{\text {turb }}^{2}} \sim 10 \times d \times \kappa \sim 10 \times \frac{d}{R}$, where $R$ is the curvature radius. Here we are interested in making order-of-magnitude estimations, and the effect of projection on the pressure is neglected. Therefore we have $p_{\text {wind }} \sim p_{\text {envelope }}$. In our calculation, $R$ is approximately the size of the outflow and $d$ is the thickness of the outflow. Here, we take advantage of the fact that $\rho_{\text {outflow }} \sim \rho_{\text {envelope }}$ and $v_{\text {outflow }} \sim 3 v_{\text {envelope. }}$. It can be seen that if $d \sim R$, centrifugal force will play an important role in changing the shape of the outflow cavity. In our case, since $d \ll R$ (Fig. 6, note that the thickness of the outflow has been artificially scaled up for clarity, and in fact our outflow layer is very thin compared with the size of the outflow), the influence of centrifugal force is within a few percent and is therefore generally insignificant.

Our case is different from the case of Canto \& Rodriguez (1980) and Biro et al. (1993) who found the centrifugal force to be important. This is because our entrainment process conserves momentum and at the same time increases the mass of the outflow significantly, and in Canto \& Rodriguez (1980) and Biro et al. (1993) the entrainment process is insignificant. To illustrate the effect of mass growth on the centrifugal force, we consider a particle of mass $m$ rotating along a circle of radius $R$ with velocity $v$. The centrifugal force can be expressed as

$f=\frac{m v^{2}}{R}=\frac{p^{2}}{m R}$

where $p$ is magnitude of the momentum of the particle. Here, it is quite clear that when the momentum is conserved, the larger the mass, the weaker the centrifugal force.

In our case, the entrainment process conserves momentum, but the mass of the outflow has been increased by a huge factor. Assuming pressure balance $p_{\text {wind }} \sim p_{\text {envelope }}$, we have $\rho_{\text {wind }} v_{\text {wind }}^{2} \sim \rho_{\text {envelope }} \sigma_{\text {envelope }}^{2}$. The mass from the wind is $m_{\text {wind }} \sim$ $\rho_{\text {wind }} v_{\text {wind }} \beta \times \mathrm{d} s$, where $\mathrm{d} s$ represents the surface area, and the mass from the envelope is $m_{\text {envelope }} \sim \alpha \times \rho_{\text {envelope }} \sigma_{\text {envelope }} \times \mathrm{d} s$. The ratio $m_{\text {envelope }} / m_{\text {wind }}$ is

$$
\begin{aligned}
\frac{m_{\text {envelope }}}{m_{\text {wind }}} & \sim \frac{\rho_{\text {envelope }} \times v_{\text {envelope }}}{\rho_{\text {wind }} \times v_{\text {wind }}} \\
& \sim \frac{p_{\text {envelope }} / \sigma_{\text {envelope }} \times \alpha}{p_{\text {wind }} / v_{\text {wind }} \times \beta} \sim \frac{1}{3} \times \frac{v_{\text {wind }}}{\sigma_{\text {envelope }}} .
\end{aligned}
$$

Since $v_{\text {wind }} \gg \sigma_{\text {envelope }}$, the mass growth from the entrainment process is huge, therefore the centrifugal force becomes insignificant.

As the wind gas mixes with the ambient gas, the momentum of the system is conserved, but the mass of the system becomes much larger, therefore the centrifugal force becomes much smaller. This is because the momentum-conserving entrainment process increases the outflow mass significantly so that the centrifugal force is insignificant.

\section{References}

Alatalo, K., Blitz, L., Young, L. M., et al. 2011, ApJ, 735, 88

Arce, H. G., \& Sargent, A. I. 2006, ApJ, 646, 1070

Arce, H. G., Shepherd, D., Gueth, F., et al. 2007, Protostars and Planets V (Tucson: University of Arizona Press), 245

Bachiller, R., \& Perez Gutierrez, M. 1997, ApJ, 487, L93

Ballesteros-Paredes, J., Klessen, R. S., Mac Low, M.-M., \& Vazquez-Semadeni,

E. 2007, Protostars and Planets V (Tucson: University of Arizona Press), 63 Bell, J. H., \& Mehta, R. D. 1990, AIAA Journal, 28, 2034

Beuther, H., \& Shepherd, D. 2005, in Cores to Clusters: Star Formation with Next Generation Telescopes, eds. M. S. N. Kumar, M. Tafalla, \& P. Caselli (New York: Springer), 324, 105

Beuther, H., Schilke, P., Menten, K. M., et al. 2002, ApJ, 566, 945

Beuther, H., Schilke, P., \& Gueth, F. 2004, ApJ, 608, 330 
Biro, S., Canto, J., Raga, A. C., \& Binette, L. 1993, Rev. Mex. Astron. Astrofis., 25,95

Brinch, C., \& Hogerheijde, M. R. 2010, A\&A, 523, A25

Cabrit, S., \& Bertout, C. 1986, ApJ, 307, 313

Cabrit, S., \& Bertout, C. 1990, ApJ, 348, 530

Cabrit, S., \& Bertout, C. 1992, A\&A, 261, 274

Canto, J. 1980, A\&A, 86, 327

Canto, J., \& Raga, A. C. 1991, ApJ, 372, 646

Canto, J., \& Rodriguez, L. F. 1980, ApJ, 239, 982

Champagne, F. H., Pao, Y. H., \& Wygnanski, I. J. 1976, J. Fluid Mech., 74, 209

Churchwell, E. 1997, ApJ, 479, L59

Cunningham, A., Frank, A., \& Hartmann, L. 2005, ApJ, 631, 1010

Cyganowski, C. J., Brogan, C. L., Hunter, T. R., Churchwell, E., \& Zhang, Q. 2011, ApJ, 729, 124

Dunham, M. M., Evans, N. J., Bourke, T. L., et al. 2010, ApJ, 721, 995

Fiege, J. D., \& Henriksen, R. N. 1996, MNRAS, 281, 1038

Keto, E., \& Zhang, Q. 2010, MNRAS, 406, 102

Krumholz, M. R., \& McKee, C. F. 2005, ApJ, 630, 250

Lada, C. J. 1985, ARA\&A, 23, 267

Larson, R. B. 1981, MNRAS, 194, 809

Lee, C.-F., Mundy, L. G., Reipurth, B., Ostriker, E. C., \& Stone, J. M. 2000, ApJ, 542, 925

Lee, C.-F., Stone, J. M., Ostriker, E. C., \& Mundy, L. G. 2001, ApJ, 557, 429

Lee, C. W., Bourke, T. L., Myers, P. C., et al. 2009, ApJ, 693, 1290

Lery, T., Henriksen, R. N., \& Fiege, J. D. 1999, A\&A, 350, 254

Li, Z.-Y., \& Shu, F. H. 1996, ApJ, 472, 211

Longmore, S. N., Pillai, T., Keto, E., Zhang, Q., \& Qiu, K. 2011, ApJ, 726, 97

Matzner, C. D. 2007, ApJ, 659, 1394

Matzner, C. D., \& McKee, C. F. 1999, ApJ, 526, L109

McKee, C. F., \& Holliman, II, J. H. 1999, ApJ, 522, 313
McKee, C. F., \& Tan, J. C. 2002, Nature, 416, 59

McKee, C. F., \& Tan, J. C. 2003, ApJ, 585, 850

McLaughlin, D. E., \& Pudritz, R. E. 1996, ApJ, 469, 194

Mueller, K. E., Shirley, Y. L., Evans, II, N. J., \& Jacobson, H. R. 2002, ApJS, 143,469

Najita, J. R., \& Shu, F. H. 1994, ApJ, 429, 808

Nakamura, F., \& Li, Z.-Y. 2007, ApJ, 662, 395

Offner, S. S. R., Lee, E. J., Goodman, A. A., \& Arce, H. 2011, ApJ, 743, 91

Ostriker, E. C. 1997, ApJ, 486, 291

Parkin, E. R., Pittard, J. M., Hoare, M. G., Wright, N. J., \& Drake, J. J. 2009, MNRAS, 400, 629

Plume, R., Jaffe, D. T., Evans, II, N. J., Martin-Pintado, J., \& Gomez-Gonzalez, J. 1997, ApJ, 476, 730

Qiu, K., Zhang, Q., \& Menten, K. M. 2011, ApJ, 728, 6

Qiu, K., Zhang, Q., Wu, J., \& Chen, H.-R. 2009, ApJ, 696, 66

Ren, J. Z., Liu, T., Wu, Y., \& Li, L. 2011, MNRAS, 415, L49

Reynolds, O. 1895, Roy. Soc. London Philos. Trans. Ser. A, 186, 123

Rogers, M. M., \& Moser, R. D. 1994, Phys. Fluids, 6, 903

San Jose-Garcia, I., Mottram, J. C., Kristensen, L. E., et al. 2013, A\&A, 553, A125

Shepherd, D. S., Watson, A. M., Sargent, A. I., \& Churchwell, E. 1998, ApJ, 507,861

Shu, F. H., Ruden, S. P., Lada, C. J., \& Lizano, S. 1991, ApJ, 370, L31

Shu, F. H., Najita, J., Ostriker, E. C., \& Shang, H. 1995, ApJ, 455, L155

Stahler, S. W. 1994, ApJ, 422, 616

Tsai, A.-L., Matsushita, S., Kong, A. K. H., Matsumoto, H., \& Kohno, K. 2012, ApJ, 752, 38

van der Tak, F. F. S., Black, J. H., Schöier, F. L., Jansen, D. J., \& van Dishoeck, E. F. 2007, A\&A, 468, 627

Watson, C., Zweibel, E. G., Heitsch, F., \& Churchwell, E. 2004, ApJ, 608, 274 